\title{
Transversity Signals in Two-Hadron Production at COMPASS
}

\author{
Christian Schill on behalf of the COMPASS collaboration
}

Physikalisches Institut der Albert-Ludwigs-Universität Freiburg Hermann-Herder-Str. 3, 79104 Freiburg, Germany.

\begin{abstract}
New results on single spin asymmetries of identified charged pion and kaon pairs produced in deep-inelastic scattering of muons on a transversely polarized ${ }^{6} \mathrm{LiD}$ target are presented. The data were taken in the years 2003 and 2004 with the COMPASS spectrometer at CERN with a $160 \mathrm{GeV}$ muon beam from the CERN SPS accelerator. The asymmetries can be interpreted in the context of transversity as a convolution of the chiral-odd interference fragmentation function $H_{1}^{\varangle}$ with the transverse spin distribution of quarks $\Delta_{T} q(x)$. The measured azimuthal target spin asymmetries on the deuteron are compatible with zero within a small statistical error of about $1 \%$.
\end{abstract}

\section{Introduction}

An important missing piece in our understanding of the spin structure of the nucleon is the transversity distribution function $\Delta_{T} q(x)$. It is the only one of the three leading-twist quark distribution functions $q(x), \Delta q(x)$ and $\Delta_{T} q(x)$ that so-far remains unmeasured. The function $\Delta_{T} q(x)$ describes the distribution of transversely polarized quarks in a transversely polarized nucleon. It is difficult to measure $\Delta_{T} q(x)$, since it is a chiral-odd function which can only be probed in combination with another chiral-odd function. So far, attempts were made to access transversity in convolution with the Collins fragmentation function in single hadron production $[2,3]$.

An alternative probe suggested to access transversity is the measurement of two-hadron production in semi-inclusive deep-inelastic scattering on a transversely polarized target. In this case, transversity is accessible via the chiral-odd two-hadron interference fragmentation function $H_{1}^{\varangle}\left(z, M_{h}^{2}\right)$. The properties of interference fragmentation functions are described in detail in Refs. $[4,5,6,7,8,9]$. New COMPASS results for identified pion and kaon pairs are presented in this contribution [1].

\section{Two-hadron asymmetry}

At leading twist, the fragmentation function of a polarized quark into a pair of hadrons is expected to be of the form

$$
D_{q}^{2 h}\left(z, M_{h}^{2}\right)+H_{1}^{\varangle}\left(z, M_{h}^{2}\right) \sin \theta \sin \phi_{R S},
$$

where $M_{h}$ is the invariant mass of the hadron pair and $z=z_{1}+z_{2}$ is the fraction of available energy carried by the two hadrons. $D_{q}^{2 h}\left(z, M_{h}^{2}\right)$ is the unpolarized fragmentation function into two hadrons, and the interference fragmentation function $H_{1}^{\varangle}\left(z, M_{h}^{2}\right)$ is the spin dependent T-odd part of the fragmentation function of a transversely polarized quark $q$ into a hadron pair. The angles $\theta$ and $\phi_{R S}$ (see Fig. 1) are defined according to Ref. [10]. $\phi_{R S}=$ $\phi_{R}+\phi_{S}-\pi$ is the sum of the azimuthal angle $\phi_{R}$ of a plane containing the two hadrons and the azimuthal angle $\phi_{S}$ of target spin vector with respect to the lepton scattering plane. In COMPASS $\phi_{R}$ is defined as the azimuthal angle of $\vec{R}_{T}$, the transverse component of the vector 


$$
\vec{R}=\frac{z_{2} \cdot \vec{p}_{1}-z_{1} \vec{p}_{2}}{z_{1}+z_{2}}
$$

where the indices 1 and 2 refer to the two final state hadrons. $\theta$ is the polar angle of the first hadron in the two-hadron centerof-mass frame with respect to the direction of the summed hadron momentum $\vec{p}_{h}=$ $\vec{p}_{1}+\vec{p}_{2}$. With the applied cuts, $\theta$ peaks close to $\pi / 2$ with $\langle\sin \theta>\approx 0.95$. The following results are obtained by integrating over $\sin \theta$. The number of hadron pairs in a bin of the Bjorken variable $x$, or of $z$, or of

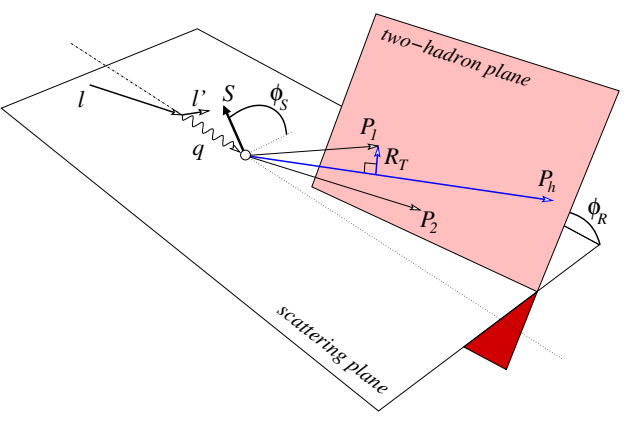

Figure 1: Definition of azimuthal angles $\phi_{R}$ and $\phi_{S}$.

$M_{h}$ is given by

$$
N^{ \pm}\left(\phi_{R S}\right)=N_{0} \cdot\left(1 \pm A_{U T}^{\sin \phi_{R S}} \cdot \sin \phi_{R S}\right),
$$

where \pm refers to the transverse target spin orientation and $N_{0}$ is the mean number of detected hadron pairs averaged over $\sin \phi_{R S}$. From the angular distribution of the hadron pairs, one can thus measure the asymmetry

$$
A_{R S}=\frac{1}{f P_{T} D} \cdot A_{U T}^{\sin \phi_{R S}},
$$

where $f \approx 0.38$ is the target dilution factor, $P_{T} \approx 0.5$ the target polarization and $D$ the depolarization factor given by $D=(1-y) /\left(1-y+y^{2} / 2\right)$, where $y$ is the fractional energy transfer of the lepton.

The measured asymmetry can be factorized into a convolution of the transversity distribution $\Delta_{T} q(x)$ of the quarks of flavor $q$ and the interference fragmentation $H_{1}^{\varangle}\left(z, M_{h}^{2}\right)$ [10]:

$$
A_{R S}=\frac{\Sigma_{q} e_{q}^{2} \cdot \Delta_{T} q(x) \cdot H_{1}^{\varangle}\left(z, M_{h}^{2}\right)}{\Sigma_{q} e_{q}^{2} \cdot q(x) \cdot D_{q}^{2 h}\left(z, M_{h}^{2}\right)},
$$

summed over all quark flavors $q$. Both the interference fragmentation function $H_{1}^{\varangle}\left(z, M_{h}^{2}\right)$ and the corresponding spin averaged fragmentation function into two hadrons $D_{q}^{2 h}\left(z, M_{h}^{2}\right)$ are unknown, and need to be measured in $e^{+} e^{-}$annihilation or to be evaluated using models $[6,7,8,9]$. They are expected to depend on $z=z_{1}+z_{2}$ and on the invariant mass $M_{h}$ of the two hadrons.

\section{Event selection}

The data discussed here have been taken in the years 2003 and 2004 in the COMPASS experiment at CERN [3]. It uses a secondary $160 \mathrm{GeV} \mu^{+}$beam from $\pi$ and $K$ decay in the CERN SPS M2 beamline. The muons are scattered on a transversely polarized solid state ${ }^{6} \mathrm{LiD}$ target. The target consists of two $60 \mathrm{~cm}$ long target cells with opposite polarization. To minimize systematic effects, the direction of the target polarization is reversed in both cells once a week. The scattered muons and the produced hadrons are detected in a $50 \mathrm{~m}$ long large-acceptance forward spectrometer with excellent particle identification capabilities. A large scale Ring Imaging Cherenkov detector (RICH) is used to distinguish pions, kaons and 
protons [11]. It allows to separate pions and kaons over a large momentum range from the Cherenkov thresholds of $3 \mathrm{GeV} / \mathrm{c}$ respectively $9 \mathrm{GeV} / \mathrm{c}$ for pions and kaons up to $43 \mathrm{GeV} / \mathrm{c}$.

The event selection was done in the same way as in the previous analysis of the Collins and Sivers asymmetries $[2,3]$. For the selection of the DIS event sample, kinematic cuts of the squared four-momentum transfer $Q^{2}>1(\mathrm{GeV} / \mathrm{c})^{2}$, the hadronic invariant mass $W>$ $5 \mathrm{GeV} / \mathrm{c}^{2}$ and the fractional energy transfer of the muon $0.1<y<0.9$ were applied. The average $x, y$ and $Q^{2}$ in the final data sample is $x=0.035, y=0.33$ and $Q^{2}=2.4(\mathrm{GeV} / \mathrm{c})^{2}$. The mean hadron multiplicity is 1.9 hadrons per event.

Hadron pairs originating from the primary vertex are selected, where the first hadron has positive and the second one negative charge. The hadron pairs are separated into $\pi^{+} \pi^{-}, K^{+} \pi^{-}, \pi^{+} K^{-}$, and $K^{+} K^{-}$pairs based on the information of the RICH detector. Selection cuts of $z_{1}>0.1$ and $z_{2}>0.1$ suppress hadrons from the target fragmentation and $z=z_{1}+z_{2}<0.9$ reject exclusively produced $\rho$-mesons. The resulting event sample contains 3.7 million $\pi^{+} \pi^{-}, 0.3$ million $K^{+} \pi^{-}, 0.25$ million $\pi^{+} K^{-}$and 0.1 million $K^{+} K^{-}$ pairs. By combining data from both target cells as well as from sub-periods with opposite target polarization in a double ratio product described in detail in Ref. [3], the acceptance function of the spectrometer cancels out and the azimuthal asymmetry $A_{R S}\left(x, z, M_{h}\right)$ is extracted by a fit to the data. In various studies, it could be shown that systematic effects of the measurement are considerably smaller than the statistical uncertainty of the data.

\section{Results}

Figure 2 shows the preliminary results for the target single spin asymmetry $A_{R S}\left(x, y, M_{h}\right)$ for identified hadron pairs. In the first row, the asymmetry for $\pi^{+} \pi^{-}$is displayed, in the second for $K^{+} \pi^{-}$pairs, in the third for $\pi^{+} K^{-}$and in the last row for $K^{+} K^{-}$pairs. The asymmetries are plotted as a function of $x, z$ and $M_{h}$. The measured asymmetries are very small and compatible with zero within the statistical precision of the data points. They do not show a significant dependence on the kinematic variables $x$ and $z$ and on the hadron pair invariant mass $M_{h}$.

\section{Discussion}

In several theoretical models, predictions have been made for the measured asymmetries $A_{R S}\left(x, z, M_{h}\right)$ for pions or unidentified hadrons on a deuteron target $[7,9]$. The expected values of the asymmetry are generally small and below $1 \%$. The small signal is attributed in these calculations to a partial cancellation of the asymmetries originating from scattering on up and down quarks of the proton and neutron in the isoscalar deuteron target. In 2007, COMPASS is taking data with a transversely polarized proton target, where the asymmetries are expected to be larger [9]. Together with the deuteron data presented here, a separation of the asymmetries originating from up and down quarks shall then be possible.

\section{References}

[1] Slides: http://indico. cern.ch/contributionDisplay $\cdot$ py? contribId=165\&sessionId=4\&conf $I d=9499$

[2] A. Bressan (COMPASS) and A. Kotzinian (COMPASS), these proceedings.

[3] V.Yu. Alexakhin et al. [COMPASS collaboration] Phys. Rev. Lett. 94, 202002 (2005) and E.S. Ageev et al. [COMPASS collaboration] Nucl. Phys. B765, 31 (2007).

[4] J.R. Collins, S.F. Heppelmann and G.A. Ladinsky, Nucl. Phys. B420, 565 (1994). 

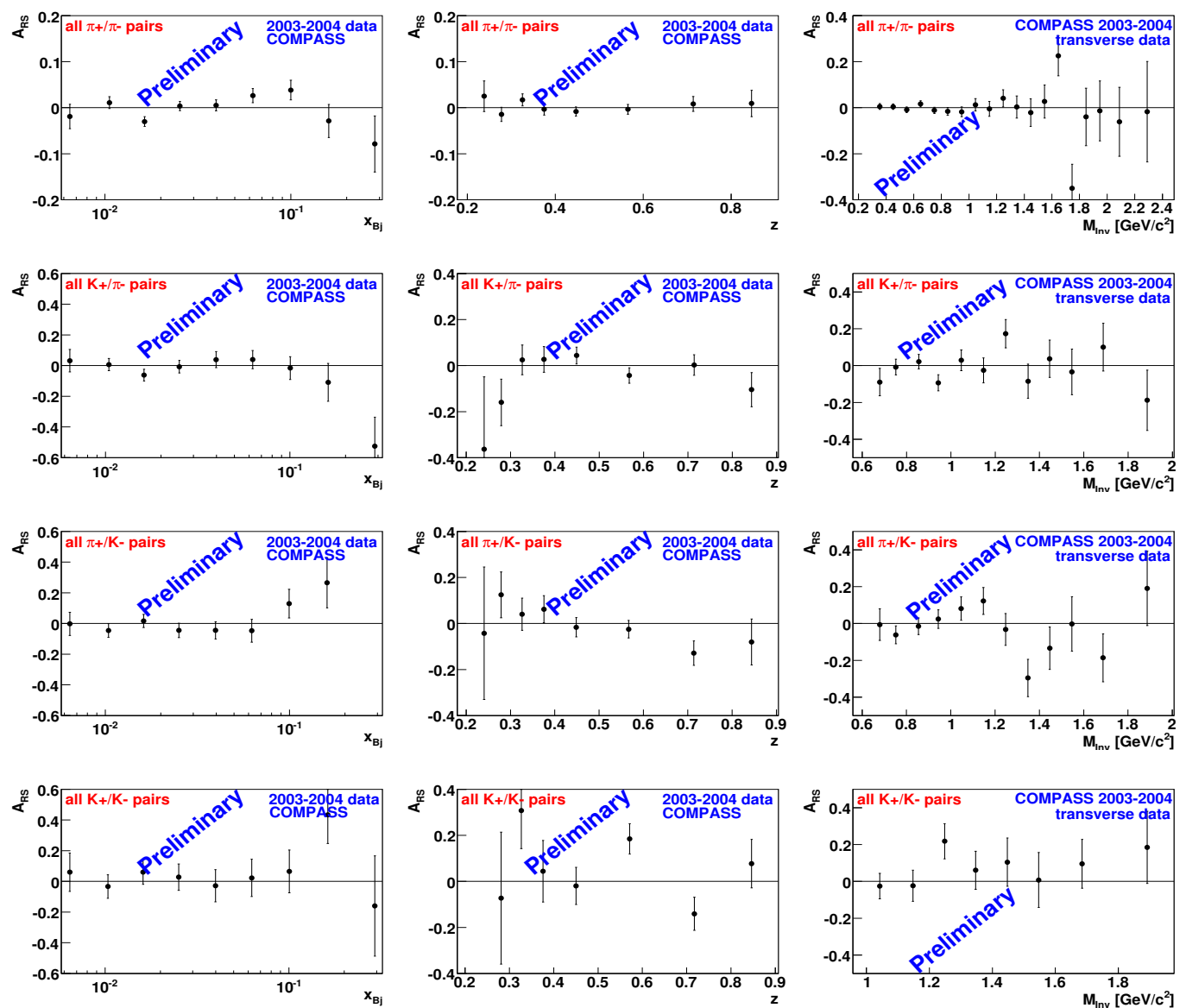

Figure 2: Asymmetries $A_{R S}$ for identified hadron pairs: $\pi^{+} \pi^{-}$(top row), $K^{+} \pi^{-}$(second row), $K^{-} \pi^{+}$(third row) and $K^{+} K^{-}$(last row). The asymmetries are shown as a function of $x$ (left colum), $z$ (middle column) and $M_{h}$ (right column).

[5] X. Artru and J.C. Collins, Z. Phys. C69, 277 (1996).

[6] R.L. Jaffe, X. Ji and J. Tang, Phys. Rev. Lett. 80, 1166 (1998).

[7] A. Bianconi, S. Boffi, R. Jakob and M. Radici, Phys. Rev. D62, 034008 (2000).

[8] M. Radici, R. Jakob and A. Bianconi, Phys. Rev. Lett D65, 074031 (2002).

[9] A. Bacchetta and M. Radici, Phys. Rev. D67, 094002 (2003), Phys. Rev. D69, 074026 (2004) and Phys. Rev. D74 114007 (2006).

[10] X. Artru, hep-ph/0207309 (2002).

[11] P. Abbon et al. [COMPASS collaboration] hep-ex/0703049, accepted by NIM A. 